\title{
Organometallics
}

\section{Synthesis and Structure of 1,3,5-Tris(diorganohydroxysilyl)benzenes. Novel Building Blocks in Supramolecular Silanol Chemistry}

\author{
Jens Beckmann, ${ }^{* a}$ Andrew Duthie, ${ }^{a}$ Gregor Reeske, ${ }^{\mathrm{a}, \mathrm{b}}$ and Markus Schürmann ${ }^{\mathrm{b}}$ \\ ${ }^{a}$ Centre for Chiral and Molecular Technologies, Deakin University, Geelong 3217, Australia. \\ ${ }^{\mathrm{b}}$ Lehrstuhl für Anorganische Chemie II, Universität Dortmund, D-44221 Dortmund, Germany.
}

\section{Supporting Information}

Table S1. Atomic coordinates and isotropic displacement parameters (in $\AA^{2}$ ) for TMSB.

Table S2. Anisotropic displacement parameters (in $\AA^{2}$ ) for TMSB.

Table S3. Selected geometric parameters $\left(\AA{ }^{\circ}\right)$ for TMSB.

Table S4. Atomic coordinates and isotropic displacement parameters (in $\AA^{2}$ ) for TPSB $\cdot \mathrm{H}_{2} \mathrm{O}$.

Table S5. Anisotropic displacement parameters (in $\AA^{2}$ ) for TPSB $\cdot \mathrm{H}_{2} \mathrm{O}$.

Table S6. Selected geometric parameters $\left(\AA{ }^{\circ}\right)$ for TPSB $\cdot \mathrm{H}_{2} \mathrm{O}$.

\footnotetext{
*Fax: 6135227 1040; Tel: 6135227 1416; E-mail: beckmann@ deakin.edu.au
} 
Table S1. Atomic coordinates and isotropic displacement parameters (in $\AA^{2}$ ) for TMSB.

\begin{tabular}{|c|c|c|c|c|c|}
\hline Atom & Wyck. & $\mathbf{x}$ & $\mathbf{y}$ & $\mathbf{z}$ & $\mathbf{U}$ \\
\hline Sil & $2 i$ & $-0.12050(6)$ & $-0.30822(6)$ & $0.43631(5)$ & \\
\hline $\mathrm{Si} 2$ & $2 i$ & $-0.31790(7)$ & $-0.53881(6)$ & $-0.15726(5)$ & \\
\hline $\mathrm{Si} 3$ & $2 i$ & $0.27720(7)$ & $0.06551(6)$ & $0.21626(5)$ & \\
\hline $\mathrm{O} 1$ & $2 i$ & $-0.26591(19)$ & $-0.28316(18)$ & $0.46420(15)$ & \\
\hline H1 & $2 i$ & $-0.345(2)$ & $-0.337(2)$ & $0.408(2)$ & $0.038(8)$ \\
\hline $\mathrm{O} 2$ & $2 i$ & $-0.43040(18)$ & $-0.49426(18)$ & $-0.25298(15)$ & \\
\hline $\mathrm{H} 2$ & $2 i$ & $-0.392(3)$ & $-0.423(2)$ & $-0.257(2)$ & $0.031(7)$ \\
\hline $\mathrm{O} 3$ & $2 i$ & $0.27367(18)$ & $0.22389(17)$ & $0.27917(16)$ & \\
\hline $\mathrm{H} 3$ & $2 i$ & $0.270(3)$ & $0.248(3)$ & $0.343(2)$ & $0.034(8)$ \\
\hline $\mathrm{C} 1$ & $2 i$ & $-0.0894(2)$ & $-0.2722(2)$ & $0.28183(18)$ & \\
\hline $\mathrm{C} 2$ & $2 i$ & $-0.1991(2)$ & $-0.3880(2)$ & $0.14404(19)$ & \\
\hline $\mathrm{H} 2 \mathrm{~A}$ & $2 i$ & $-0.2917(18)$ & $-0.4786(18)$ & $0.1306(15)$ & $0.012(4)$ \\
\hline $\mathrm{C} 3$ & $2 i$ & $-0.1765(2)$ & $-0.3751(2)$ & $0.02435(18)$ & \\
\hline $\mathrm{C} 4$ & $2 i$ & $-0.0347(2)$ & $-0.2354(2)$ & $0.04883(19)$ & \\
\hline $\mathrm{H} 4 \mathrm{~A}$ & $2 i$ & $-0.0160(19)$ & $-0.2236(18)$ & $-0.0289(16)$ & $0.018(5)$ \\
\hline C5 & $2 i$ & $0.0802(2)$ & $-0.1146(2)$ & $0.18282(18)$ & \\
\hline C6 & $2 i$ & $0.0484(2)$ & $-0.1368(2)$ & $0.29785(19)$ & \\
\hline H6A & $2 i$ & $0.1305(19)$ & $-0.0542(19)$ & $0.3908(16)$ & $0.016(4)$ \\
\hline C11 & $2 i$ & $0.0707(3)$ & $-0.1667(3)$ & $0.6019(2)$ & \\
\hline H11A & $2 i$ & $0.159(3)$ & $-0.176(2)$ & $0.5925(18)$ & $0.040(6)$ \\
\hline H11B & $2 i$ & $0.099(2)$ & $-0.070(2)$ & $0.619(2)$ & $0.043(7)$ \\
\hline $\mathrm{H} 11 \mathrm{C}$ & $2 i$ & $0.057(2)$ & $-0.184(2)$ & $0.682(2)$ & $0.061(7)$ \\
\hline $\mathrm{C} 21$ & $2 i$ & $-0.1899(3)$ & $-0.5154(3)$ & $0.3945(3)$ & \\
\hline $\mathrm{H} 21 \mathrm{~A}$ & $2 i$ & $-0.293(3)$ & $-0.581(2)$ & $0.316(2)$ & $0.049(7)$ \\
\hline H21B & $2 i$ & $-0.205(2)$ & $-0.544(2)$ & $0.467(2)$ & $0.048(6)$ \\
\hline $\mathrm{H} 21 \mathrm{C}$ & $2 i$ & $-0.110(3)$ & $-0.532(2)$ & $0.373(2)$ & $0.050(7)$ \\
\hline C31 & $2 i$ & $-0.4725(3)$ & $-0.7173(3)$ & $-0.1511(3)$ & \\
\hline H31A & $2 i$ & $-0.416(3)$ & $-0.757(2)$ & $-0.103(2)$ & $0.053(7)$ \\
\hline H31B & $2 i$ & $-0.540(3)$ & $-0.696(2)$ & $-0.107(2)$ & $0.050(7)$ \\
\hline $\mathrm{H} 31 \mathrm{C}$ & $2 i$ & $-0.551(2)$ & $-0.803(2)$ & $-0.245(2)$ & $0.048(6)$ \\
\hline C41 & $2 i$ & $-0.1892(3)$ & $-0.5757(3)$ & $-0.2414(3)$ & \\
\hline $\mathrm{H} 41 \mathrm{~A}$ & $2 i$ & $-0.256(3)$ & $-0.663(2)$ & $-0.334(2)$ & $0.050(7)$ \\
\hline H41B & $2 i$ & $-0.118(2)$ & $-0.487(2)$ & $-0.2486(19)$ & $0.038(6)$ \\
\hline $\mathrm{H} 41 \mathrm{C}$ & $2 i$ & $-0.126(3)$ & $-0.602(2)$ & $-0.185(2)$ & $0.049(7)$ \\
\hline C51 & $2 i$ & $0.3028(4)$ & $0.0558(4)$ & $0.0506(3)$ & \\
\hline H51A & $2 i$ & $0.310(3)$ & $-0.028(3)$ & $0.016(2)$ & $0.054(8)$ \\
\hline H51B & $2 i$ & $0.217(3)$ & $0.050(3)$ & $-0.013(2)$ & $0.059(8)$ \\
\hline $\mathrm{H} 51 \mathrm{C}$ & $2 i$ & $0.402(3)$ & $0.147(3)$ & $0.072(2)$ & $0.063(8)$ \\
\hline C61 & $2 i$ & $0.4515(3)$ & $0.0840(3)$ & $0.3485(3)$ & \\
\hline H61A & $2 i$ & $0.440(3)$ & $0.095(3)$ & $0.433(2)$ & $0.062(8)$ \\
\hline H61B & $2 i$ & $0.455(3)$ & $-0.007(3)$ & $0.317(3)$ & $0.083(10)$ \\
\hline H61C & $2 i$ & $0.552(3)$ & $0.167(3)$ & $0.367(2)$ & $0.069(8)$ \\
\hline
\end{tabular}


Table S2. Anisotropic displacement parameters (in $\AA^{2}$ ) for TMSB.

$\begin{array}{lllllll}\text { Atom } & \boldsymbol{U}_{\mathbf{1 1}} & \boldsymbol{U}_{\mathbf{2 2}} & \boldsymbol{U}_{\mathbf{3 3}} & \boldsymbol{U}_{\mathbf{1 2}} & \boldsymbol{U}_{\mathbf{1 3}} & \boldsymbol{U}_{\mathbf{2 3}} \\ \mathrm{S} \text { 1 } & 0.0238(3) & 0.0260(4) & 0.0208(3) & 0.0101(3) & 0.0093(2) & 0.0110(3) \\ \text { Si2 } & 0.0249(3) & 0.0236(3) & 0.0221(3) & 0.0105(3) & 0.0084(2) & 0.0104(3) \\ \text { Si3 } & 0.0286(3) & 0.0215(4) & 0.0265(3) & 0.0087(3) & 0.0140(3) & 0.0107(3) \\ \text { O1 } & 0.0229(9) & 0.0345(10) & 0.0225(8) & 0.0116(8) & 0.0081(7) & 0.0082(8) \\ \text { O2 } & 0.0268(8) & 0.0238(9) & 0.0324(8) & 0.0083(7) & 0.0061(6) & 0.0153(8) \\ \text { O3 } & 0.0432(10) & 0.0271(9) & 0.0272(9) & 0.0162(7) & 0.0215(8) & 0.0148(8) \\ \text { C1 } & 0.0219(11) & 0.0212(12) & 0.0226(10) & 0.011(1) & 0.0098(9) & 0.0087(10) \\ \text { C2 } & 0.0205(11) & 0.0214(12) & 0.0298(11) & 0.0093(10) & 0.0127(10) & 0.0141(11) \\ \text { C3 } & 0.0218(11) & 0.0227(12) & 0.024(1) & 0.0126(10) & 0.0105(9) & 0.0114(10) \\ \text { C4 } & 0.0294(12) & 0.0305(13) & 0.0236(11) & 0.017(1) & 0.0146(10) & 0.0159(11) \\ \text { C5 } & 0.0250(11) & 0.0209(12) & 0.0244(10) & 0.0121(10) & 0.0117(9) & 0.0111(10) \\ \text { C6 } & 0.0252(12) & 0.0201(12) & 0.0223(11) & 0.0106(10) & 0.0091(10) & 0.0062(10) \\ \text { C11 } & 0.0287(13) & 0.0377(17) & 0.0289(13) & 0.0142(12) & 0.011(1) & 0.0140(13) \\ \text { C21 } & 0.0448(17) & 0.0369(16) & 0.0369(14) & 0.0208(14) & 0.0184(14) & 0.0226(13) \\ \text { C31 } & 0.0378(14) & 0.0262(14) & 0.0352(14) & 0.0122(12) & 0.0140(12) & 0.0148(13) \\ \text { C41 } & 0.0412(15) & 0.0425(17) & 0.0319(14) & 0.0219(14) & 0.0151(12) & 0.0123(14) \\ \text { C51 } & 0.0496(18) & 0.0365(17) & 0.0368(15) & 0.0134(15) & 0.0268(14) & 0.0147(14) \\ \text { C61 } & 0.0313(15) & 0.0375(17) & 0.0537(17) & 0.0129(14) & 0.0157(13) & 0.0200(14)\end{array}$


Table S3. Selected geometric parameters $\left(\AA{ }^{\circ}{ }^{\circ}\right)$ for TMSB.

\begin{tabular}{|c|c|c|c|}
\hline $\mathrm{Si} 1-\mathrm{O} 1$ & $1.653(2)$ & $\mathrm{C} 3-\mathrm{C} 2$ & $1.397(3)$ \\
\hline Si $1-C 11$ & $1.849(4)$ & $\mathrm{C} 3-\mathrm{C} 4$ & $1.403(3)$ \\
\hline $\mathrm{Si} 1-\mathrm{C} 21$ & $1.851(3)$ & $\mathrm{C} 3-\mathrm{Si} 2$ & $1.867(4)$ \\
\hline Si1-C1 & $1.875(2)$ & $\mathrm{C} 4-\mathrm{C} 5$ & $1.390(3)$ \\
\hline $\mathrm{Si} 2-\mathrm{O} 2$ & $1.647(2)$ & $\mathrm{C} 4-\mathrm{C} 3$ & $1.403(3)$ \\
\hline $\mathrm{Si} 2-\mathrm{C} 41$ & $1.846(4)$ & $\mathrm{C} 5-\mathrm{C} 4$ & $1.390(3)$ \\
\hline $\mathrm{Si} 2-\mathrm{C} 31$ & $1.851(3)$ & $\mathrm{C} 5-\mathrm{C} 6$ & $1.407(3)$ \\
\hline $\mathrm{Si} 2-\mathrm{C} 3$ & $1.867(4)$ & $\mathrm{C} 5-\mathrm{Si} 3$ & $1.874(3)$ \\
\hline $\mathrm{Si} 3-\mathrm{O} 3$ & $1.645(2)$ & $\mathrm{C} 6-\mathrm{C} 1$ & $1.392(3)$ \\
\hline $\mathrm{Si} 3-\mathrm{C} 61$ & $1.850(4)$ & $\mathrm{C} 6-\mathrm{C} 5$ & $1.407(3)$ \\
\hline $\mathrm{Si} 3-\mathrm{C} 51$ & $1.851(4)$ & $\mathrm{C} 11-\mathrm{Si} 1$ & $1.849(4)$ \\
\hline $\mathrm{Si} 3-\mathrm{C} 5$ & $1.874(3)$ & $\mathrm{C} 21-\mathrm{Si} 1$ & $1.851(3)$ \\
\hline $\mathrm{O} 1-\mathrm{Si} 1$ & $1.653(2)$ & $\mathrm{C} 31-\mathrm{Si} 2$ & $1.851(3)$ \\
\hline $\mathrm{O} 2-\mathrm{Si} 2$ & $1.647(2)$ & $\mathrm{C} 41-\mathrm{Si} 2$ & $1.846(4)$ \\
\hline $\mathrm{O} 3-\mathrm{Si} 3$ & $1.645(2)$ & $\mathrm{C} 51-\mathrm{Si} 3$ & $1.851(4)$ \\
\hline $\mathrm{C} 1-\mathrm{C} 6$ & $1.392(3)$ & $\mathrm{C} 61-\mathrm{Si} 3$ & $1.850(4)$ \\
\hline $\mathrm{C} 1-\mathrm{C} 2$ & $1.395(3)$ & $\mathrm{O} 1-\mathrm{H} 1$ & $0.701(14)$ \\
\hline $\mathrm{C} 1-\mathrm{Si} 1$ & $1.875(2)$ & $\mathrm{O} 2-\mathrm{H} 2$ & $0.694(21)$ \\
\hline $\mathrm{C} 2-\mathrm{C} 1$ & $1.395(3)$ & $\mathrm{O} 3-\mathrm{H} 3$ & $0.684(25)$ \\
\hline $\mathrm{C} 2-\mathrm{C} 3$ & $1.397(3)$ & & \\
\hline $\mathrm{O} 1-\mathrm{Si} 1-\mathrm{C} 11$ & $105.47(12)$ & $\mathrm{C} 61-\mathrm{Si} 3-\mathrm{C} 51$ & $112.38(13)$ \\
\hline $\mathrm{O} 1-\mathrm{Si} 1-\mathrm{C} 21$ & $108.10(13)$ & $\mathrm{C} 61-\mathrm{Si} 3-\mathrm{C} 5$ & $107.43(12)$ \\
\hline $\mathrm{O} 1-\mathrm{Si} 1-\mathrm{C} 1$ & 111.69(9) & $\mathrm{C} 51-\mathrm{Si} 3-\mathrm{C} 5$ & $111.42(14)$ \\
\hline $\mathrm{C} 11-\mathrm{Si} 1-\mathrm{C} 21$ & $112.72(13)$ & $\mathrm{C} 6-\mathrm{C} 1-\mathrm{C} 2$ & $116.90(18)$ \\
\hline $\mathrm{C} 11-\mathrm{Si} 1-\mathrm{C} 1$ & $111.29(8)$ & $\mathrm{C} 6-\mathrm{C} 1-\mathrm{Si} 1$ & $122.51(15)$ \\
\hline $\mathrm{C} 21-\mathrm{Si} 1-\mathrm{C} 1$ & $107.59(12)$ & $\mathrm{C} 2-\mathrm{C} 1-\mathrm{Si} 1$ & $120.40(13)$ \\
\hline $\mathrm{O} 2-\mathrm{Si} 2-\mathrm{C} 41$ & $109.99(13)$ & $\mathrm{C} 1-\mathrm{C} 2-\mathrm{C} 3$ & $123.69(16)$ \\
\hline $\mathrm{O} 2-\mathrm{Si} 2-\mathrm{C} 31$ & $104.57(12)$ & $\mathrm{C} 2-\mathrm{C} 3-\mathrm{C} 4$ & $116.15(18)$ \\
\hline $\mathrm{O} 2-\mathrm{Si} 2-\mathrm{C} 3$ & $111.13(8)$ & $\mathrm{C} 2-\mathrm{C} 3-\mathrm{Si} 2$ & $122.32(13)$ \\
\hline $\mathrm{C} 41-\mathrm{Si} 2-\mathrm{C} 31$ & $111.91(15)$ & $\mathrm{C} 4-\mathrm{C} 3-\mathrm{Si} 2$ & $121.41(14)$ \\
\hline $\mathrm{C} 41-\mathrm{Si} 2-\mathrm{C} 3$ & $108.35(11)$ & $\mathrm{C} 5-\mathrm{C} 4-\mathrm{C} 3$ & $123.56(18)$ \\
\hline $\mathrm{C} 31-\mathrm{Si} 2-\mathrm{C} 3$ & $110.89(11)$ & $\mathrm{C} 4-\mathrm{C} 5-\mathrm{C} 6$ & $116.76(16)$ \\
\hline $\mathrm{O} 3-\mathrm{Si} 3-\mathrm{C} 61$ & $109.15(12)$ & $\mathrm{C} 4-\mathrm{C} 5-\mathrm{Si} 3$ & $123.50(15)$ \\
\hline $\mathrm{O} 3-\mathrm{Si} 3-\mathrm{C} 51$ & $105.60(13)$ & $\mathrm{C} 6-\mathrm{C} 5-\mathrm{Si} 3$ & $119.62(15)$ \\
\hline $\mathrm{O} 3-\mathrm{Si} 3-\mathrm{C} 5$ & $110.88(9)$ & $\mathrm{C} 1-\mathrm{C} 6-\mathrm{C} 5$ & $122.93(19)$ \\
\hline
\end{tabular}


Table S4. Atomic coordinates and isotropic displacement parameters (in $\AA^{2}$ ) for TPSB $\cdot \mathrm{H}_{2} \mathrm{O}$.

\begin{tabular}{|c|c|c|c|c|c|}
\hline Atom & Wyck. & $\mathbf{x}$ & $\mathbf{y}$ & $\mathbf{z}$ & $\mathbf{U}$ \\
\hline $\mathrm{C} 1$ & $4 e^{\circ}$ & $0.3403(2)$ & $0.10066(11)$ & $0.56015(14)$ & \\
\hline $\mathrm{C} 2$ & $4 e$ & $0.4372(2)$ & $0.13295(12)$ & $0.53231(15)$ & \\
\hline $\mathrm{H} 2 \mathrm{~A}$ & $4 e$ & 0.52050 & 0.11880 & 0.55030 & 0.0330 \\
\hline C3 & $4 e$ & $0.4142(2)$ & $0.18532(12)$ & $0.47885(15)$ & \\
\hline $\mathrm{C} 4$ & $4 e$ & $0.28781(19)$ & $0.20575(11)$ & $0.45394(14)$ & \\
\hline $\mathrm{H} 4 \mathrm{~A}$ & $4 e$ & 0.27040 & 0.24030 & 0.41800 & 0.0310 \\
\hline C5 & $4 e$ & $0.18599(19)$ & $0.17706(12)$ & $0.48009(15)$ & \\
\hline C6 & $4 e$ & $0.2154(2)$ & $0.12505(12)$ & $0.53280(15)$ & \\
\hline H6A & $4 e$ & 0.14960 & 0.10520 & 0.55110 & 0.0330 \\
\hline $\mathrm{C} 11$ & $4 e$ & $0.2770(2)$ & $0.02734(14)$ & $0.69793(15)$ & \\
\hline $\mathrm{C} 12$ & $4 e$ & $0.2725(2)$ & $0.08556(15)$ & $0.73960(18)$ & \\
\hline $\mathrm{H} 12 \mathrm{~A}$ & $4 e$ & 0.31120 & 0.12410 & 0.72590 & 0.0580 \\
\hline C13 & $4 e$ & $0.2126(3)$ & $0.08856(17)$ & $0.8010(2)$ & \\
\hline H13A & $4 e$ & 0.21220 & 0.12850 & 0.82810 & 0.0760 \\
\hline C14 & $4 e$ & $0.1535(2)$ & $0.03216(18)$ & $0.82162(18)$ & \\
\hline H14A & $4 e$ & 0.11320 & 0.03360 & 0.86280 & 0.0650 \\
\hline C15 & $4 e$ & $0.1548(2)$ & $-0.02630(17)$ & $0.78073(19)$ & \\
\hline $\mathrm{H} 15 \mathrm{~A}$ & $4 e$ & 0.11440 & -0.06440 & 0.79410 & 0.0600 \\
\hline $\mathrm{C} 16$ & $4 e$ & $0.2158(2)$ & $-0.02885(14)$ & $0.71966(17)$ & \\
\hline H16A & $4 e$ & 0.21590 & -0.06890 & 0.69270 & 0.0530 \\
\hline $\mathrm{C} 21$ & $4 e$ & $0.5428(2)$ & $0.01436(13)$ & $0.66452(17)$ & \\
\hline $\mathrm{C} 22$ & $4 e$ & $0.6234(2)$ & $-0.01651(14)$ & $0.62688(18)$ & \\
\hline $\mathrm{H} 22 \mathrm{~A}$ & $4 e$ & 0.59170 & -0.03330 & 0.57790 & 0.0750 \\
\hline $\mathrm{C} 23$ & $4 e$ & $0.7529(3)$ & $-0.02394(16)$ & $0.6593(2)$ & \\
\hline $\mathrm{H} 23 \mathrm{~A}$ & $4 e$ & 0.80560 & -0.04630 & 0.63250 & 0.0940 \\
\hline $\mathrm{C} 24$ & $4 e$ & $0.8005(3)$ & $0.0013(2)$ & $0.7292(2)$ & \\
\hline $\mathrm{H} 24 \mathrm{~A}$ & $4 e$ & 0.88620 & -0.00390 & 0.75150 & 0.1010 \\
\hline $\mathrm{C} 25$ & $4 e$ & $0.7231(3)$ & $0.0344(2)$ & $0.7671(2)$ & \\
\hline $\mathrm{H} 25 \mathrm{~A}$ & $4 e$ & 0.75630 & 0.05360 & 0.81470 & 0.1580 \\
\hline $\mathrm{C} 26$ & $4 e$ & $0.5937(3)$ & $0.03982(19)$ & $0.7349(2)$ & \\
\hline $\mathrm{H} 26 \mathrm{~A}$ & $4 e$ & 0.54100 & 0.06140 & 0.76230 & 0.1150 \\
\hline C31 & $4 e$ & $0.5847(2)$ & $0.30879(12)$ & $0.48345(15)$ & \\
\hline C32 & $4 e$ & $0.7070(2)$ & $0.33523(14)$ & $0.49239(16)$ & \\
\hline $\mathrm{H} 32 \mathrm{~A}$ & $4 e$ & 0.76940 & 0.30980 & 0.47680 & 0.0470 \\
\hline C33 & $4 e$ & $0.7381(3)$ & $0.39804(15)$ & $0.52372(18)$ & \\
\hline $\mathrm{H} 33 \mathrm{~A}$ & $4 e$ & 0.82070 & 0.41400 & 0.52960 & 0.0630 \\
\hline C34 & $4 e$ & $0.6478(3)$ & $0.43673(15)$ & $0.54612(19)$ & \\
\hline $\mathrm{H} 34 \mathrm{~A}$ & $4 e$ & 0.66830 & 0.47930 & 0.56670 & 0.0800 \\
\hline $\mathrm{C} 35$ & $4 e$ & $0.5256(3)$ & $0.41213(15)$ & $0.5380(2)$ & \\
\hline H35A & $4 e$ & 0.46380 & 0.43810 & 0.55340 & 0.0800 \\
\hline C36 & $4 e$ & $0.4953(2)$ & $0.34964(14)$ & $0.50724(17)$ & \\
\hline H36A & $4 e$ & 0.41250 & 0.33400 & 0.50210 & 0.0570 \\
\hline C41 & $4 e$ & $0.4743(2)$ & $0.23775(15)$ & $0.33357(16)$ & \\
\hline $\mathrm{C} 42$ & $4 e$ & $0.4450(3)$ & $0.29880(16)$ & $0.3002(2)$ & \\
\hline $\mathrm{H} 42 \mathrm{~A}$ & $4 e$ & 0.46410 & 0.33720 & 0.33030 & 0.0840 \\
\hline $\mathrm{C} 43$ & $4 e$ & $0.3877(4)$ & $0.3063(2)$ & $0.2230(2)$ & \\
\hline $\mathrm{H} 43 \mathrm{~A}$ & $4 e$ & 0.37030 & 0.34910 & 0.20250 & 0.1180 \\
\hline $\mathrm{C} 44$ & $4 e$ & $0.3568(3)$ & $0.2514(2)$ & $0.1770(2)$ & \\
\hline $\mathrm{H} 44 \mathrm{~A}$ & $4 e$ & 0.31800 & 0.25600 & 0.12540 & 0.0860 \\
\hline $\mathrm{C} 45$ & $4 e$ & $0.3842(3)$ & $0.1901(2)$ & $0.2087(2)$ & \\
\hline $\mathrm{H} 45 \mathrm{~A}$ & $4 e$ & 0.36420 & 0.15180 & 0.17850 & 0.0880 \\
\hline $\mathrm{C} 46$ & $4 e$ & $0.4412(3)$ & $0.18327(16)$ & $0.2850(2)$ & \\
\hline $\mathrm{H} 46 \mathrm{~A}$ & $4 e$ & 0.45830 & 0.14020 & 0.30480 & 0.0740 \\
\hline C51 & $4 e$ & $0.04602(19)$ & $0.30539(13)$ & $0.47618(17)$ & \\
\hline C52 & $4 e$ & $0.0688(2)$ & $0.32106(14)$ & $0.55343(17)$ & \\
\hline $\mathrm{H} 52 \mathrm{~A}$ & $4 e$ & 0.06180 & 0.28730 & 0.58830 & 0.0440 \\
\hline $\mathrm{C} 53$ & $4 e$ & $0.1015(2)$ & $0.38521(16)$ & $0.58083(18)$ & \\
\hline $\mathrm{H} 53 \mathrm{~A}$ & $4 e$ & 0.11630 & 0.39420 & 0.63320 & 0.0580 \\
\hline C54 & $4 e$ & $0.1119(2)$ & $0.43556(15)$ & $0.5296(2)$ & \\
\hline H54A & $4 e$ & 0.13570 & 0.47870 & 0.54720 & 0.0580 \\
\hline C55 & $4 e$ & $0.0870(3)$ & $0.42195(15)$ & $0.4522(2)$ & \\
\hline $\mathrm{H} 55 \mathrm{~A}$ & $4 e$ & 0.09150 & 0.45630 & 0.41750 & 0.0640 \\
\hline C56 & $4 e$ & $0.0550(2)$ & $0.35710(15)$ & $0.42550(18)$ & \\
\hline H56A & $4 e$ & 0.03950 & 0.34840 & 0.37310 & 0.0510 \\
\hline
\end{tabular}




$\begin{array}{llllll}\mathrm{C} 61 & 4 e & -0.0308(2) & 0.21270(12) & 0.33998(15) & \\ \mathrm{C} 62 & 4 e & 0.0468(2) & 0.20529(14) & 0.28723(18) & \\ \mathrm{H} 62 \mathrm{~A} & 4 e & 0.13260 & 0.19600 & 0.30540 & 0.0580 \\ \mathrm{C} 63 & 4 e & 0.0011(2) & 0.21119(15) & 0.20881(18) & \\ \mathrm{H} 63 \mathrm{~A} & 4 e & 0.05610 & 0.20670 & 0.17540 & 0.0680 \\ \mathrm{C} 64 & 4 e & -0.1259(3) & 0.22372(14) & 0.18027(17) & \\ \mathrm{H} 64 \mathrm{~A} & 4 e & -0.15710 & 0.22820 & 0.12770 & 0.0580 \\ \mathrm{C} 65 & 4 e & -0.2062(2) & 0.22950(14) & 0.23031(18) & \\ \mathrm{H} 65 \mathrm{~A} & 4 e & -0.29240 & 0.23700 & 0.21150 & 0.0560 \\ \mathrm{C} 66 & 4 e & -0.1592(2) & 0.22425(13) & 0.30844(17) & \\ \mathrm{H} 66 \mathrm{~A} & 4 e & -0.21510 & 0.22860 & 0.34130 & 0.0450 \\ \mathrm{O} 1 & 4 e & 0.32768(13) & -0.04330(8) & 0.56685(10) & \\ \mathrm{H} 1 & 4 e & 0.26790 & -0.03380 & 0.53160 & 0.0490 \\ \mathrm{O} 2 & 4 e & 0.66915(12) & 0.17892(8) & 0.45261(10) & \\ \mathrm{H} 2 & 4 e & 0.65190 & 0.14120 & 0.46590 & 0.0430 \\ \mathrm{O} 3 & 4 e & -0.07025(12) & 0.17570(8) & 0.49108(9) & \\ \mathrm{H} 3 & 4 e & -0.14230 & 0.19100 & 0.47760 & 0.0410 \\ \mathrm{O} 4 & 4 e & -0.0937(3) & 0.04293(17) & 0.5299(3) & \\ \mathrm{Si} 1 & 4 e & 0.36958(6) & 0.02409(4) & 0.62155(5) & \\ \mathrm{Si} 2 & 4 e & 0.53979(6) & 0.22634(4) & 0.43747(5) & \\ \mathrm{Si} 3 & 4 e & 0.02620(6) & 0.21553(4) & 0.44558(5) & \\ \mathrm{H} 4 & 4 e & -0.078(3) & 0.0895(17) & 0.526(2) & 0.150(18) \\ \mathrm{H} 5 & 4 e & -0.063(3) & 0.0232(19) & 0.503(3) & 0.09(2)\end{array}$


Table S5. Anisotropic displacement parameters $\left(i n \AA^{2}\right.$ ) for TPSB $\cdot \mathrm{H}_{2} \mathrm{O}$.

\begin{tabular}{|c|c|c|c|c|c|c|}
\hline Atom & $U_{11}$ & $U_{22}$ & $U_{33}$ & $U_{12}$ & $U_{13}$ & $U_{23}$ \\
\hline $\mathrm{C} 1$ & $.0235(15)$ & $0.0230(17)$ & $0.030(2)$ & $0.0017(13)$ & $0.0072(13)$ & $-0.0016(14)$ \\
\hline $\mathrm{C} 2$ & $0.0166(15)$ & $0.0276(18)$ & $0.038(2)$ & $0.0057(13)$ & $0.0033(13)$ & $-0.0053(16)$ \\
\hline C3 & $0.0175(14)$ & $0.0248(18)$ & $0.035(2)$ & $0.0019(12)$ & $0.0057(13)$ & $-0.0014(15)$ \\
\hline $\mathrm{C} 4$ & $0.0244(14)$ & $0.0244(17)$ & $0.0281(19)$ & $0.0032(13)$ & $0.0047(12)$ & $0.0031(14)$ \\
\hline C5 & $0.0211(14)$ & $0.0233(17)$ & $0.0288(19)$ & $0.0003(13)$ & $0.0078(13)$ & $-0.0006(15)$ \\
\hline C6 & $0.0223(15)$ & $0.0295(18)$ & $0.032(2)$ & $-0.0049(13)$ & $0.0101(13)$ & $-0.0025(15)$ \\
\hline C11 & $0.0285(15)$ & $0.0245(18)$ & $0.040(2)$ & $0.0001(14)$ & $0.0094(14)$ & $0.0031(17)$ \\
\hline $\mathrm{C} 12$ & $0.0511(19)$ & $0.049(2)$ & $0.052(3)$ & $-0.0087(16)$ & $0.0280(19)$ & $-0.001(2)$ \\
\hline $\mathrm{C} 13$ & $0.072(2)$ & $0.062(3)$ & $0.065(3)$ & $-0.011(2)$ & $0.035(2)$ & $-0.009(2)$ \\
\hline C14 & $0.0405(18)$ & $0.071(3)$ & $0.054(3)$ & $0.0020(19)$ & $0.0191(17)$ & $0.014(2)$ \\
\hline C15 & $0.0372(18)$ & $0.054(3)$ & $0.060(3)$ & $-0.0004(17)$ & $0.0159(18)$ & $0.023(2)$ \\
\hline $\mathrm{C} 16$ & $0.0406(18)$ & $0.032(2)$ & $0.060(3)$ & $0.0036(15)$ & $0.0116(17)$ & $0.0080(19)$ \\
\hline $\mathrm{C} 21$ & $0.0291(16)$ & $0.0312(19)$ & $0.037(2)$ & $0.0045(14)$ & $0.0087(15)$ & $0.0058(16)$ \\
\hline $\mathrm{C} 22$ & $0.0367(19)$ & $0.070(3)$ & $0.080(3)$ & $0.0007(17)$ & $0.0144(19)$ & $-0.037(2)$ \\
\hline $\mathrm{C} 23$ & $0.037(2)$ & $0.078(3)$ & $0.125(4)$ & $0.014(2)$ & $0.028(2)$ & $-0.019(3)$ \\
\hline $\mathrm{C} 24$ & $0.031(2)$ & $0.144(4)$ & $0.071(3)$ & 0.01 & -0.00 & $0.021(3)$ \\
\hline $\mathrm{C} 25$ & $0.046(2)$ & $0.286(6)$ & $0.055(3)$ & 0.02 & -0.00 & -0.03 \\
\hline $\mathrm{C} 26$ & $0.041(2)$ & $0.187(4)$ & $0.056(3)$ & 0.02 & $0.0010(19)$ & -0.03 \\
\hline $\mathrm{C} 31$ & $0.0276(15)$ & $0.0233(18)$ & $0.035(2)$ & $0.0028(13)$ & $0.0059(14)$ & 0.002 \\
\hline $\mathrm{C} 32$ & $0.0361(18)$ & $0.025(2)$ & $4(2)$ & $-0.0015(14)$ & $0.0043(15)$ & -0.00 \\
\hline C33 & 0.04 & 0.03 & $0.066(3)$ & (17) & $-0.0026(18)$ & 0.00 \\
\hline C34 & $0.094(3)$ & $0.033(2)$ & $0.072(3)$ & & $0.017(2)$ & $-0.018(2)$ \\
\hline C35 & $0.082(3)$ & $0.037(2)$ & $0.095(3)$ & -0.00 & $0.048(2)$ & -0.01 \\
\hline C36 & $0.0483(19)$ & $0.029(2)$ & $0.071(3)$ & $-0.0056(16)$ & 0.025 & $-0.0076(18)$ \\
\hline C41 & $0.0258(16)$ & $0.033(2)$ & $0.038(2)$ & $-0.0050(14)$ & 0.014 & $0.0019(18)$ \\
\hline C42 & $0.110(3)$ & $0.050(3)$ & $0.039(3)$ & $-0.017(2)$ & $-0.005(2)$ & $0.002(2)$ \\
\hline C43 & $0.160(4)$ & $0.068(3)$ & $0.050(3)$ & $-0.015(3)$ & $-0.012(3)$ & $0.014(3)$ \\
\hline C44 & $0.075(3)$ & $0.098(4)$ & $0.039(3)$ & -0.00 & $0.005(2)$ & $0.010(3)$ \\
\hline C45 & $0.097(3)$ & $0.077(3)$ & $0.043(3)$ & $-0.018(2)$ & $0.011(2)$ & $-0.019(2)$ \\
\hline C46 & $0.086(2)$ & $0.050(3)$ & $0.046(3)$ & $0.002(2)$ & $0.009(2)$ & $-0.002(2)$ \\
\hline C51 & $0.0165(14)$ & $0.0288(19)$ & $0.031(2)$ & $0.0024(12)$ & $0.0045(13)$ & $0.0057(16)$ \\
\hline C52 & $0.0388(17)$ & $0.028(2)$ & $0.039(2)$ & $-0.0017(14)$ & $-0.0019(15)$ & $0.0044(17)$ \\
\hline C53 & $0.0475(19)$ & $0.046(2)$ & $0.044(2)$ & $-0.0027(16)$ & $-0.0047(17)$ & $-0.004(2)$ \\
\hline C54 & $0.0511(19)$ & $0.030(2)$ & $0.062(3)$ & $-0.0034(15)$ & $0.009(2)$ & $-0.003(2)$ \\
\hline C55 & $0.071(2)$ & $0.031(2)$ & $0.060(3)$ & $-0.0044(17)$ & $0.018(2)$ & $0.012(2)$ \\
\hline C56 & $0.0496(19)$ & $0.033(2)$ & $0.046(2)$ & $0.0010(15)$ & $0.0109(16)$ & $-0.0011(19)$ \\
\hline C61 & $0.0237(15)$ & $0.0299(18)$ & $0.040(2)$ & $-0.0003(13)$ & $0.0081(14)$ & $0.0010(16)$ \\
\hline C62 & $0.0300(16)$ & $0.071(3)$ & $0.042(2)$ & $-0.0002(15)$ & $0.0052(17)$ & $-0.0072(19)$ \\
\hline C63 & $0.0409(19)$ & $0.093(3)$ & $0.040(3)$ & $-0.0116(18)$ & $0.0145(17)$ & $-0.009(2)$ \\
\hline C64 & $0.054(2)$ & $0.059(2)$ & $0.029(2)$ & $-0.0091(18)$ & $0.0005(17)$ & $-0.0031(18)$ \\
\hline C65 & $0.0400(18)$ & $0.050(2)$ & $0.043(3)$ & $0.0017(16)$ & $-0.0026(18)$ & $-0.0032(19)$ \\
\hline C66 & $0.0320(17)$ & $0.043(2)$ & $0.038(2)$ & $0.0011(14)$ & $0.0076(15)$ & $-0.0014(17)$ \\
\hline O1 & $0.0382(10)$ & $0.0292(13)$ & $0.0501(15)$ & $0.0033(9)$ & $-0.0016(9)$ & $-0.004(1)$ \\
\hline $\mathrm{O} 2$ & $0.0226(9)$ & $0.0211(11)$ & $0.0653(15)$ & $-0.0005(8)$ & $0.0143(9)$ & $0.0085(10)$ \\
\hline $\mathrm{O} 3$ & $0.0177(9)$ & $0.0334(12)$ & $0.0516(14)$ & $0.0017(8)$ & $0.0089(9)$ & $0.0068(10)$ \\
\hline $\mathrm{O} 4$ & $0.0459(15)$ & $0.040(2)$ & $0.182(4)$ & $-0.0102(15)$ & $-0.015(2)$ & $0.023(2)$ \\
\hline Sil & $0.0283(4)$ & $0.0285(5)$ & $0.0404(6)$ & $0.0009(4)$ & $0.0065(4)$ & $0.0009(5)$ \\
\hline $\mathrm{Si} 2$ & $0.0211(4)$ & $0.0285(5)$ & $0.0417(6)$ & $-0.0002(4)$ & $0.0087(4)$ & $0.0002(5)$ \\
\hline $\mathrm{Si} 3$ & $0.0198(4)$ & $0.0306(5)$ & $0.0375(6)$ & $0.0000(4)$ & $0.0051(4)$ & $0.0013(5)$ \\
\hline
\end{tabular}


Table S6. Selected geometric parameters $\left(\stackrel{\AA}{\AA}{ }^{\circ}\right)$ for TPSB $\cdot \mathrm{H}_{2} \mathrm{O}$.

\begin{tabular}{|c|c|c|c|}
\hline $\mathrm{C} 1-\mathrm{C} 2$ & $1.404(5)$ & $\mathrm{C} 42-\mathrm{C} 41$ & $1.359(4)$ \\
\hline $\mathrm{C} 1-\mathrm{C} 6$ & $1.415(5)$ & $\mathrm{C} 42-\mathrm{C} 43$ & $1.391(7)$ \\
\hline $\mathrm{C} 1-\mathrm{Si} 1$ & $1.863(3)$ & $\mathrm{C} 43-\mathrm{C} 44$ & $1.364(6)$ \\
\hline $\mathrm{C} 2-\mathrm{C} 3$ & $1.398(4)$ & $\mathrm{C} 43-\mathrm{C} 42$ & $1.391(7)$ \\
\hline $\mathrm{C} 2-\mathrm{C} 1$ & $1.404(5)$ & $\mathrm{C} 44-\mathrm{C} 45$ & $1.350(6)$ \\
\hline $\mathrm{C} 3-\mathrm{C} 2$ & $1.398(4)$ & $\mathrm{C} 44-\mathrm{C} 43$ & $1.364(6)$ \\
\hline $\mathrm{C} 3-\mathrm{C} 4$ & $1.399(4)$ & $\mathrm{C} 45-\mathrm{C} 44$ & $1.350(6)$ \\
\hline $\mathrm{C} 3-\mathrm{Si} 2$ & $1.862(5)$ & $\mathrm{C} 45-\mathrm{C} 46$ & $1.374(7)$ \\
\hline $\mathrm{C} 4-\mathrm{C} 3$ & $1.399(4)$ & $\mathrm{C} 46-\mathrm{C} 45$ & $1.374(7)$ \\
\hline $\mathrm{C} 4-\mathrm{C} 5$ & $1.402(4)$ & $\mathrm{C} 46-\mathrm{C} 41$ & $1.385(5)$ \\
\hline $\mathrm{C} 5-\mathrm{C} 6$ & $1.388(4)$ & C51-C52 & $1.382(5)$ \\
\hline $\mathrm{C} 5-\mathrm{C} 4$ & $1.402(4)$ & C51-C56 & $1.386(4)$ \\
\hline $\mathrm{C} 5-\mathrm{Si} 3$ & $1.864(5)$ & $\mathrm{C} 51-\mathrm{Si} 3$ & $1.868(3)$ \\
\hline $\mathrm{C} 6-\mathrm{C} 5$ & $1.388(4)$ & $\mathrm{C} 52-\mathrm{C} 51$ & $1.382(5)$ \\
\hline $\mathrm{C} 6-\mathrm{C} 1$ & $1.415(5)$ & $\mathrm{C} 52-\mathrm{C} 53$ & $1.385(4)$ \\
\hline $\mathrm{C} 11-\mathrm{C} 12$ & $1.382(4)$ & C53-C54 & $1.376(5)$ \\
\hline $\mathrm{C} 11-\mathrm{C} 16$ & $1.394(4)$ & $\mathrm{C} 53-\mathrm{C} 52$ & $1.385(4)$ \\
\hline $\mathrm{C} 11-\mathrm{Si} 1$ & $1.853(6)$ & $\mathrm{C} 54-\mathrm{C} 55$ & $1.375(5)$ \\
\hline $\mathrm{C} 12-\mathrm{C} 11$ & $1.382(4)$ & $\mathrm{C} 54-\mathrm{C} 53$ & $1.376(5)$ \\
\hline $\mathrm{C} 12-\mathrm{C} 13$ & $1.386(6)$ & $\mathrm{C} 55-\mathrm{C} 54$ & $1.375(5)$ \\
\hline $\mathrm{C} 13-\mathrm{C} 14$ & $1.378(5)$ & $\mathrm{C} 55-\mathrm{C} 56$ & $1.393(4)$ \\
\hline $\mathrm{C} 13-\mathrm{C} 12$ & $1.386(6)$ & $\mathrm{C} 56-\mathrm{C} 51$ & $1.386(4)$ \\
\hline $\mathrm{C} 14-\mathrm{C} 15$ & $1.374(5)$ & $\mathrm{C} 56-\mathrm{C} 55$ & $1.393(4)$ \\
\hline $\mathrm{C} 14-\mathrm{C} 13$ & $1.378(5)$ & C61-C62 & $1.393(6)$ \\
\hline $\mathrm{C} 15-\mathrm{C} 14$ & $1.374(5)$ & C61-C66 & $1.396(5)$ \\
\hline $\mathrm{C} 15-\mathrm{C} 16$ & $1.388(6)$ & $\mathrm{C} 61-\mathrm{Si} 3$ & $1.852(6)$ \\
\hline $\mathrm{C} 16-\mathrm{C} 15$ & $1.388(6)$ & C62-C63 & $1.384(6)$ \\
\hline $\mathrm{C} 16-\mathrm{C} 11$ & $1.394(4)$ & C62-C61 & $1.393(6)$ \\
\hline $\mathrm{C} 21-\mathrm{C} 22$ & $1.353(5)$ & C63-C64 & $1.377(5)$ \\
\hline $\mathrm{C} 21-\mathrm{C} 26$ & $1.355(6)$ & C63-C62 & $1.384(6)$ \\
\hline $\mathrm{C} 21-\mathrm{Si} 1$ & $1.870(6)$ & C64-C65 & $1.374(6)$ \\
\hline $\mathrm{C} 22-\mathrm{C} 21$ & $1.353(5)$ & C64-C63 & $1.377(5)$ \\
\hline $\mathrm{C} 22-\mathrm{C} 23$ & $1.399(6)$ & C65-C64 & $1.374(6)$ \\
\hline $\mathrm{C} 23-\mathrm{C} 24$ & $1.340(6)$ & C65-C66 & $1.380(6)$ \\
\hline $\mathrm{C} 23-\mathrm{C} 22$ & $1.399(6)$ & C66-C65 & $1.380(6)$ \\
\hline $\mathrm{C} 24-\mathrm{C} 23$ & $1.340(6)$ & C66-C61 & $1.396(5)$ \\
\hline $\mathrm{C} 24-\mathrm{C} 25$ & $1.351(6)$ & $\mathrm{O} 1-\mathrm{Si} 1$ & $1.662(3)$ \\
\hline $\mathrm{C} 25-\mathrm{C} 24$ & $1.351(6)$ & $\mathrm{O} 2-\mathrm{Si} 2$ & $1.657(2)$ \\
\hline $\mathrm{C} 25-\mathrm{C} 26$ & $1.394(6)$ & $\mathrm{O} 3-\mathrm{Si} 3$ & $1.651(4)$ \\
\hline $\mathrm{C} 26-\mathrm{C} 21$ & $1.355(6)$ & $\mathrm{Si} 1-\mathrm{O} 1$ & $1.662(3)$ \\
\hline $\mathrm{C} 26-\mathrm{C} 25$ & $1.394(6)$ & $\mathrm{Si} 1-\mathrm{C} 11$ & $1.853(6)$ \\
\hline $\mathrm{C} 31-\mathrm{C} 36$ & $1.394(4)$ & $\mathrm{Si} 1-\mathrm{C} 1$ & $1.863(3)$ \\
\hline $\mathrm{C} 31-\mathrm{C} 32$ & $1.396(3)$ & $\mathrm{Si} 1-\mathrm{C} 21$ & $1.870(6)$ \\
\hline $\mathrm{C} 31-\mathrm{Si} 2$ & $1.851(3)$ & $\mathrm{Si} 2-\mathrm{O} 2$ & $1.657(2)$ \\
\hline $\mathrm{C} 32-\mathrm{C} 33$ & $1.380(4)$ & $\mathrm{Si} 2-\mathrm{C} 41$ & $1.850(6)$ \\
\hline $\mathrm{C} 32-\mathrm{C} 31$ & $1.396(3)$ & $\mathrm{Si} 2-\mathrm{C} 31$ & $1.851(3)$ \\
\hline $\mathrm{C} 33-\mathrm{C} 34$ & $1.365(5)$ & $\mathrm{Si} 2-\mathrm{C} 3$ & $1.862(5)$ \\
\hline $\mathrm{C} 33-\mathrm{C} 32$ & $1.380(4)$ & $\mathrm{Si} 3-\mathrm{O} 3$ & $1.651(4)$ \\
\hline $\mathrm{C} 34-\mathrm{C} 33$ & $1.365(5)$ & $\mathrm{Si} 3-\mathrm{C} 61$ & $1.852(6)$ \\
\hline C34-C35 & $1.383(5)$ & $\mathrm{Si} 3-\mathrm{C} 5$ & $1.864(5)$ \\
\hline $\mathrm{C} 35-\mathrm{C} 36$ & $1.370(4)$ & $\mathrm{Si} 3-\mathrm{C} 51$ & $1.868(3)$ \\
\hline $\mathrm{C} 35-\mathrm{C} 34$ & $1.383(5)$ & $\mathrm{O} 1-\mathrm{H} 1$ & $0.820(4)$ \\
\hline $\mathrm{C} 36-\mathrm{C} 35$ & $1.370(4)$ & $\mathrm{O} 2-\mathrm{H} 2$ & $0.820(2)$ \\
\hline $\mathrm{C} 36-\mathrm{C} 31$ & $1.394(4)$ & $\mathrm{O} 3-\mathrm{H} 3$ & $0.820(2)$ \\
\hline $\mathrm{C} 41-\mathrm{C} 42$ & $1.359(4)$ & $\mathrm{O} 4-\mathrm{H} 4$ & $0.947(34)$ \\
\hline $\mathrm{C} 41-\mathrm{C} 46$ & $1.385(5)$ & $\mathrm{O} 4-\mathrm{H} 5$ & $0.749(48)$ \\
\hline $\mathrm{C} 41-\mathrm{Si} 2$ & $1.850(6)$ & & \\
\hline $\mathrm{C} 2-\mathrm{C} 1-\mathrm{C} 6$ & $116.46(20)$ & $\mathrm{C} 41-\mathrm{C} 42-\mathrm{C} 43$ & $122.77(31)$ \\
\hline $\mathrm{C} 2-\mathrm{C} 1-\mathrm{Si} 1$ & $122.45(18)$ & $\mathrm{C} 44-\mathrm{C} 43-\mathrm{C} 42$ & $120.59(33)$ \\
\hline $\mathrm{C} 6-\mathrm{C} 1-\mathrm{Si} 1$ & $120.78(17)$ & $\mathrm{C} 45-\mathrm{C} 44-\mathrm{C} 43$ & $117.93(37)$ \\
\hline $\mathrm{C} 3-\mathrm{C} 2-\mathrm{C} 1$ & $123.04(22)$ & $\mathrm{C} 44-\mathrm{C} 45-\mathrm{C} 46$ & $121.01(33)$ \\
\hline $\mathrm{C} 2-\mathrm{C} 3-\mathrm{C} 4$ & $116.83(21)$ & $\mathrm{C} 45-\mathrm{C} 46-\mathrm{C} 41$ & $122.83(31)$ \\
\hline $\mathrm{C} 2-\mathrm{C} 3-\mathrm{Si} 2$ & $123.97(18)$ & C $52-\mathrm{C} 51-\mathrm{C} 56$ & $117.38(27)$ \\
\hline $\mathrm{C} 4-\mathrm{C} 3-\mathrm{Si} 2$ & $119.16(17)$ & $\mathrm{C} 52-\mathrm{C} 51-\mathrm{Si} 3$ & $119.46(21)$ \\
\hline
\end{tabular}




$\begin{array}{llll}\text { C3-C4-C5 } & 123.63(20) & \text { C56-C51-Si3 } & 122.64(21) \\ \text { C6-C5-C4 } & 116.55(21) & \text { C51-C52-C53 } & 122.48(27) \\ \text { C6-C5-Si3 } & 126.19(18) & \text { C54-C53-C52 } & 119.16(28) \\ \text { C4-C5-Si3 } & 117.15(16) & \text { C55-C54-C53 } & 119.77(31) \\ \text { C5-C6-C1 } & 123.46(22) & \text { C54-C55-C56 } & 120.42(30) \\ \text { C12-C11-C16 } & 116.70(25) & \text { C51-C56-C55 } & 120.75(27) \\ \text { C12-C11-Si1 } & 120.69(20) & \text { C62-C61-C66 } & 115.56(21) \\ \text { C16-C11-Si1 } & 122.49(19) & \text { C62-C61-Si3 } & 124.95(21) \\ \text { C11-C12-C13 } & 122.43(27) & \text { C66-C61-Si3 } & 119.27(19) \\ \text { C14-C13-C12 } & 119.70(29) & \text { C63-C62-C61 } & 122.51(27) \\ \text { C15-C14-C13 } & 119.32(31) & \text { C64-C63-C62 } & 119.95(25) \\ \text { C14-C15-C16 } & 120.49(27) & \text { C65-C64-C63 } & 119.29(27) \\ \text { C15-C16-C11 } & 121.35(24) & \text { C64-C65-C66 } & 120.18(27) \\ \text { C22-C21-C26 } & 116.94(28) & \text { C65-C66-C61 } & 122.46(24) \\ \text { C22-C21-Si1 } & 122.28(18) & \text { O1-Si1-C11 } & 109.74(11) \\ \text { C26-C21-Si1 } & 120.76(22) & \text { O1-Si1-C1 } & 108.91(10) \\ \text { C21-C22-C23 } & 122.15(24) & \text { O1-Si1-C21 } & 106.2(1) \\ \text { C24-C23-C22 } & 119.62(31) & \text { C11-Si1-C1 } & 110.68(12) \\ \text { C23-C24-C25 } & 119.55(34) & \text { C11-Si1-C21 } & 110.46(11) \\ \text { C24-C25-C26 } & 120.11(32) & \text { C1-Si1-C21 } & 110.74(11) \\ \text { C21-C26-C25 } & 121.56(31) & \text { O2-Si2-C41 } & 111.13(10) \\ \text { C36-C31-C32 } & 116.26(20) & \text { O2-Si2-C31 } & 107.53(10) \\ \text { C36-C31-Si2 } & 121.27(19) & \text { O2-Si2-C3 } & 110.08(9) \\ \text { C32-C31-Si2 } & 122.37(18) & \text { C41-Si2-C31 } & 110.02(13) \\ \text { C33-C32-C31 } & 121.92(25) & \text { C41-Si2-C3 } & 107.25(12) \\ \text { C34-C33-C32 } & 120.13(28) & \text { C31-Si2-C3 } & 110.86(11) \\ \text { C33-C34-C35 } & 119.47(31) & \text { O3-Si3-C61 } & 112.21(10) \\ \text { C36-C35-C34 } & 120.21(28) & \text { O3-Si3-C5 } & 105.95(9) \\ \text { C35-C36-C31 } & 122.00(26) & \text { O3-Si3-C51 } & 111.13(10) \\ \text { C42-C41-C46 } & 114.88(28) & \text { C61-Si3-C5 } & 113.90(11) \\ \text { C42-C41-Si2 } & 123.44(23) & \text { C61-Si3-C51 } & 108.56(12) \\ \text { C46-C41-Si2 } & 121.46(23) & \text { C5-Si3-C51 } & 104.87(11) \\ & & & \end{array}$

